\section{UCL}

Université

catholique

de Louvain
I NSTITUT DE STATISTIQUE

B I O S T A T I S T I Q U E E T

SCIENCES ACTUARIELLES

( I S B A )
DISCUSSION PAPER

$2018 / 15$

A self-organizing predictive map for non-life insurance 


\title{
A self-organizing predictive map for non-life insurance
}

\author{
Donatien Hainaut* \\ ISBA, Université Catholique de Louvain, Belgium
}

May 25, 2018

\begin{abstract}
This article explores the capacity of self-organizing maps (SOMs) for analysing non-life insurance data. Contrary to feed forward neural networks, also called perceptron, a SOM does not need any a priori information on the relevancy of variables. During the learning procedure, the SOM algorithm selects the most relevant combination of explanatory variables and reduces by this way the collinearity bias. However, the specific features of insurance data require adapting the classic SOM framework to manage categorical variables and the low frequency of claims. This work proposes several extensions of SOMs in order to study the claims frequency of a portfolio of motorcycle insurances.
\end{abstract}

KEYWORDS : Neural network, self organizing map, non-life insurance, claims frequency, regression models

\section{Introduction}

Among neural models, the Multilayer Perceptron (see e.g. Hertz et al. (1991) ) is one of the most popular. This model is an extension of the Rosenblatt's perceptron (1958) and is inspired by the functioning of neural cells constituting animal brains. This model admits both quantitative and categorical variables as inputs and is efficient to perform classification or non linear regression analysis. Prior to year 2010, neural networks were mainly applied to evaluate insurer's credit risk or fraud detection. E.g. Brockett et al. (1994) and (2006) train a multilayer perceptron to detect insurer insolvency. Whereas Viane et al. (2002) compare classification techniques for detection of expert automobile insurance fraud.

The use of neural networks in actuarial sciences is relatively recent. Ogunnaike and $\mathrm{Si}$ (2017) use a feed-forward neural network to predict insurance claim severity losses. Sakthivel and Rajitha (2017) compare the performance of Poisson regression models with

*Postal address: Voie du Roman Pays 20, 1348 Louvain-la-Neuve (Belgium) . E-mail to: donatien.hainaut(at)uclouvain.be 
neural networks to forecast the claims number. Wüthrich and Buser (2017) provide an excellent overview of the usefulness of these methods for actuaries. In life insurance, Hainaut (2017) proposes a perceptron-based model to forecast future mortality.

The perceptron is a method with supervised learning. It means that we have to a priori identify the most relevant variables and to know the desired outputs for combinations of these variables. For example, forecasting the frequency of car accidents with a perceptron requires an a priori segmentation of some explanatory variables like the driver's age into categories, in a similar manner to Generalized Linear Models (see e.g. Ohlsson and Johansson (2010)). The misspecification of these categories can induce a large bias in the forecast. On the other hand, the presence of collinearity between covariates affects the accuracy of the prediction. In this situation, the coefficient estimates of the multiple regression may change erratically in response to small changes in the model or the data. Self-organizing maps offer an elegant solution to segment explanatory variables and to detect dependence among covariates.

Self-organizing maps are artificial neural networks that do not ask any a priori information on the relevancy of variables. For this reason, they belong to the family of unsupervised algorithms. This method developed by Kohonen (1982) aims to analyze the original information by simplifying the amount of rough data, computing some basic features, and giving visual representation. More precisely, SOMs produce a low dimensional (typically two-dimensional), discretized representation of the input space of the training samples, called a map, and is therefore a method to perform reduction of dimensions. As perceptrons, SOMs are used for fraud detection (Brockett et al., 1998) or failure prediction (Huysmans et al. 2006).

This article contributes to the literature both from an empirical and methodological points of view. At the best of our knowledge, self organizing maps have not been applied yet to study non-life insurance data. The features of insurance data are: a low frequency of claims, a high number of policyholders and a mix of quantitative and categorical variables. These distinctive characteristics require to adapt the Kohonen's algorithm in several directions. First, we propose in section 2 a Bayesian extension of SOMs in order to regress the claims frequency on quantitative explanatory variables. Without recourse to a Bayesian assumption, it is not possible to obtain a reliable estimate of claims frequencies. Mainly because in this case the empirical frequency is null for many sub-groups of policyholders due to the low number of claims. Second, the original Kohonen's algorithm was designed to study quantitative variables. In sections 3 and 4, we propose a SOM to study categorical variables. In a similar manner to multiple correspondence analysis, we define a $\chi^{2}$ distance between combinations of categories. This approach, based on the article of Cottrel et al. (2004) allows us to regroup categorical variables and to detect dominant features in the portfolio of contracts. Finally, the theory introduced in later sections serves us to develop a SOM regressing claims frequency on quantitative and categorical variables. Empirical 
results reveal that this last algorithm is a powerful tool for segmenting policyholders.

\section{The self-organizing maps (SOM)}

Self-organizing maps offer an elegant solution to segment explanatory variables and to detect dependence among covariates. Initially, Kohonen (1982) developed SOMs in order to analyze quantitative variables. In the first subsection, we present this algorithm and apply it to an insurance data set to regroup policyholders with respect to quantitative variables. In section 2.2, we show how SOMs maybe adapted in order to regress the claims frequency on quantitative variables in a Bayesian framework.

\section{$I_{X} I$ Grid of neurons}

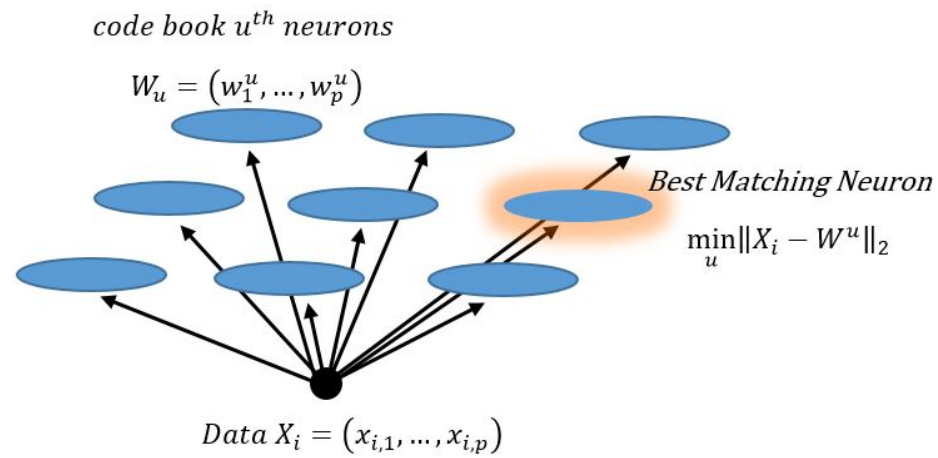

Figure 1: Illustration of the grid of neurons. The data is sent to all neurons and the best matching neuron is the only one to be activated. The best matching neuron has the closest codebook to data among all neurons.

\subsection{The SOM for quantitative variables}

In this case, we assume that features of insurance policies are exclusively quantitative variables. The number of insurance policies is denoted by $N$. Each of these policies is described by $p$ real-valued variables. The data are arranged in a table $X$ with $N$ rows and $p$

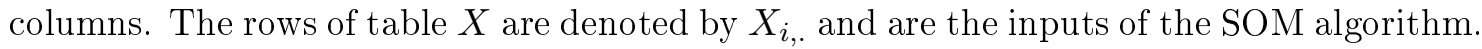

As illustrated in figure 1, the map is generally represented by a two-dimensional grid, with $l$ by $l$ nodes or neurons, indexed by $u=1, \ldots, l^{2}$. This grid is described by a $l^{2} \times 2$ matrix $C$. $C$ contains the coordinates of nodes in $\mathbb{R}^{2}$. In this article, the domain on which is defined the grid is the unit square $[0,1] \times[0,1]$. Neurons are equally spaced on this pavement. In order to define the neighbourhood of a neuron in this grid, we introduce a topological distance between neurons. Here, we use the Euclidian distance between lines $u$ and $v$ of the matrix $C$ : $\left\|C_{u}-C_{v}\right\|_{2}$. 
Algorithm 1 Kohonen's algorithm for quantitative variables.

Initialization :

Randomly attribute a vector of weights to each node of the map.

Main procedure :

For $e=0$ to maximum epoch, $e_{\max }$

For $i=1$ to $N$

1) Find the node $u$ matching at best the features of the $i^{\text {th }}$ policy

$$
u=B M N(i)=\arg \min _{u} d(u, i) .
$$

This node is the BMN (best matching node).

BMN

2) Update the weight vectors of the nodes in the neighbourhood of the

(including the BMN itself) by pulling them closer in $\mathbb{R}^{p}$ to the input

vector:

$$
W_{v}(e+1)=W_{v}(e)+\theta(u, v, e) \epsilon(e)\left(X_{i, .}-W_{v}(e)\right)
$$

where

$$
\begin{aligned}
\epsilon(e) & =\epsilon_{0}\left(\frac{e_{\max }-e}{e_{\max }}\right) \\
\theta(u, v, e) & =\theta_{0} \exp \left(-\frac{\left(\left\|C_{u}-C_{v}\right\|_{2}\right)^{2}}{2 \sigma(e)^{2}}\right) \\
\sigma(e) & =\sigma_{0}\left(\frac{1.2 e_{\max }-e}{e_{\max }}\right)
\end{aligned}
$$

End loop on policies, $i$

3) Calculation of the total distance $d^{\text {total }}$ between policies and best maching nodes:

$$
d^{\text {total }}=\sum_{i=1}^{N}\left\|W_{B M N(i)}-X_{i, .}\right\|_{2}
$$

End loop on epochs, $e$

Furthermore, we associate to each node a codebook denoted by $W_{u}=\left\{w_{1}^{u}, \ldots, w_{p}^{u}\right\}$ that is a $\mathbb{R}^{p}$ vector of weights for $u=1, \ldots, l^{2}$. We have a twofold objective. First, we aim to associate a node of the grid to each insurance policy. Second, we wish to determine codebook vectors $W_{u}$ for $u=1, \ldots, l^{2}$ that contain the average profile of policies assigned to node $u$. The vector $W_{u}$ may be seen as the barycenter or center of gravity in $\mathbb{R}^{d}$ of policies associated to the $u^{\text {th }}$ neuron. The definition of this barycenter requires the definition of a distance between the $u^{\text {th }}$ node and the $i^{\text {th }}$ insurance policy, whose respective positions in 
$\mathbb{R}^{p}$ are identified by $W_{u}$ and $X_{i, .}$. In the case of quantitative variables, we use the Euclidian distance:

$$
\begin{aligned}
d(u, i) & :=\left\|W_{u}-X_{i, .}\right\|_{2} u=1, \ldots, l^{2} i=1, \ldots, N \\
& =\sqrt{\sum_{k=1}^{p}\left(w_{k}^{u}-x_{i, k}\right)^{2}} .
\end{aligned}
$$

Remark that the neural map admits a double representation. One is on a pavement $[0,1] \times[0,1]$ and positions of neurons are fixed. And the other one is in $\mathbb{R}^{d}$ : positions of nodes are determined by the $p$-vectors $W_{u}$ for $u=1, \ldots, l^{2}$. Kohonen (1982) proposed a procedure to construct this map that is recalled in algorithm 1 . The algorithm iteratively scans the portfolio and finds for each policy the neuron with the closest codebook to its features. This neuron is called the best matching node (BMN). After this step, weights of neurons in the neighbourhood of this BMN are updated in the direction of the policy features. The size of the update is proportional to the epoch of the algorithm and to the distance between the BMN and updated neurons. Notice that functions $\epsilon(e)$ and $\sigma(e)$ in equations (3) and (5) may be replaced by any other decreasing functions of the epoch, $e$. The total distance $d^{\text {total }}$ is the error of classification if we use the feature $W_{B M N(i)}$ for the $i^{t h}$ policy, instead of the real one $X_{i, .}$. This distance is monitored to check the convergence of the algorithm. When it does not vary anymore, the learning of the neural net is finished.

The speed of convergence of the Kohonen's algorithm depends on initial weights $W_{u}(0)$. They should be chosen in order to reflect as much as possible the largest set of features of policies. The convergence also depends on parameters, $\epsilon_{0}, \theta_{0}$ and $\sigma_{0}$ of equations (3), (4) and (5). If they are too high, weights of neurons oscillate during the first iterations. If $\epsilon_{0}$, $\theta_{0}$ and $\sigma_{0}$ are too small, modifications of the codebook are not enough significant. In both case, the number of epochs must be increased to attain convergence.

To illustrate this section, we apply the Kohonen algorithm to data from the Swedish insurance company Wasa, before its fusion with Länsförsäkringar Alliance in 1999. The data set is available on the companion website of the book of Ohlsson and Johansson (2010) and contains information about motorcycles insurances over the period 1994-1998. Each policy is described by quantitative and categorical variables. The quantitative variables are the insured's age and the age of his vehicle. The categorical variables are: the policyholder's gender, the geographic zone and the category of the vehicle. The category of the vehicle is based on the ratio power in $\mathrm{KW} \times 100 /$ vehicle weight in $\mathrm{kg}+75$, rounded to the nearest integer. The database also reports the number of claims, the total claim costs and the duration of the contract for each policies. Table 1 summarizes the information provided by categorical variables. 


\begin{tabular}{ccc}
\hline Rating factors & Class & Class description \\
\hline \hline Gender & M & Male \\
& K & Female \\
\hline Geographic area & 1 & Central and semi-central parts of \\
& & Sweden's three lartgest cities \\
& 2 & Suburbs plus middle-sized cities \\
& 3 & Lesser towns, except those in 5 or 7 \\
& 4 & small towns and countryside \\
& 5 & Northen towns \\
& 6 & Northen countryside \\
\hline Vehicle class & 7 & Gotland (Sweden's largest island) \\
& 1 & EV ratio -5 \\
& 2 & EV ratio 6-8 \\
& 3 & EV ratio 9-12 \\
& 4 & EV ratio 13-15 \\
& 5 & EV ratio 16-19 \\
& 6 & EV ratio 20-24 \\
& 7 & EV ratio $25-$ \\
\hline
\end{tabular}

Table 1: Rating factors o motorcycle insurances. Source: Ohlsson and Johansson (2010).

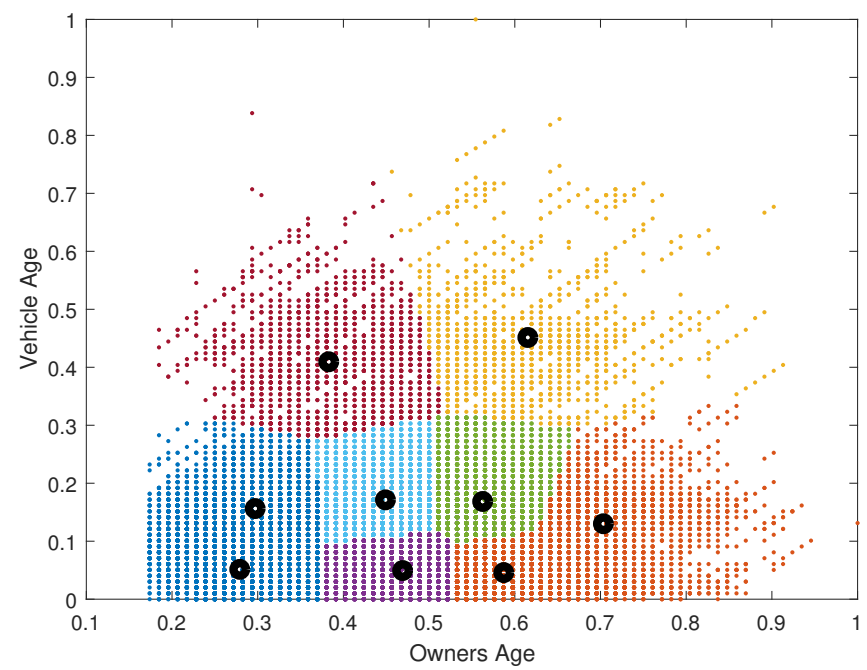

Figure 2: Kohonen's map of the portfolio, with 9 neurons. The segmenting variables are the owner's age and age of the vehicule. Each policy is represented by a dot. Black dots point out the position of neurons (the coordinates are the codebooks of neurons). The colors identify the area of influence of neurons. 
The database counts $N=62436$ policies (after removing contracts with a null duration). In this section, we focus on two quantitative variables: the owner's age and age of the vehicle. Before running the Kohonen's algorithm we scale the variables in order to convert their domain on the pavement $[0,1] \times[0,1]$. The columns of the matrix $X$ respectively contain the rescaled ages of owners and vehicles as follows

$$
x_{i, 1}=\frac{\text { Age } i^{\text {th }} \text { policyholder }}{\operatorname{Max}(\text { Age })} \quad x_{i, 2}=\frac{\text { Vehicle Age } i^{\text {th }} \text { policy }}{\text { Max }(\text { Vehicle Age })} .
$$

We build a map of the portfolio that regroups policyholders according to these two criteria. The number of epochs is $e_{\max }=100$ and the grid of neurons count 9 elements $(l=9)$. The initial codebooks are chosen in order to regularly cover the $[0,1] \times[0,1]$ pavement. The parameters of equations (3), (4) and (5) for the update of codebooks are: $\epsilon_{0}=0.01, \theta_{0}=1$ and $\sigma_{0}=0.10$. These values have been chosen by trials and errors in order to ensure a quick convergence. The figure 2 shows the Kohonen's map in the space of variables, in which policies are identified by a dot. Each colored area represents a group of policies centred around a neuron (black dots). The right graph of figure 3 reports the evolution of the total distance $d^{\text {total }}$ between policies and BMN codebooks. This distance is nearly stable and the convergence is achieved after 60 iterations. The right graph of this figure shows the number of policies associated to each neurons. Two neurons are coupled to less than $3 \%$ of policies. The others are associated to sub-groups of policies representative of $7 \%$ to $17 \%$ of the total portfolio. Table 2 reports codebooks of neurons. $w_{1}^{u} \times \max ($ Age $)$ and $w_{1}^{u} \times \max (V e h$. Age $)$ for $u=1$ to 9 are respectively the average owner's age and vehicle age of the $u^{\text {th }}$ subset of policies. An analysis of the average frequency of claims per sub-groups reveals that this frequency tends to decrease with the owner's age. However, the pooling of policies is based only on ages of policyholders and vehicles, not on claims frequency. In the next section, we modify the Kohonen's algorithm in order to delimit subsets of policies with homogeneous claims frequencies.
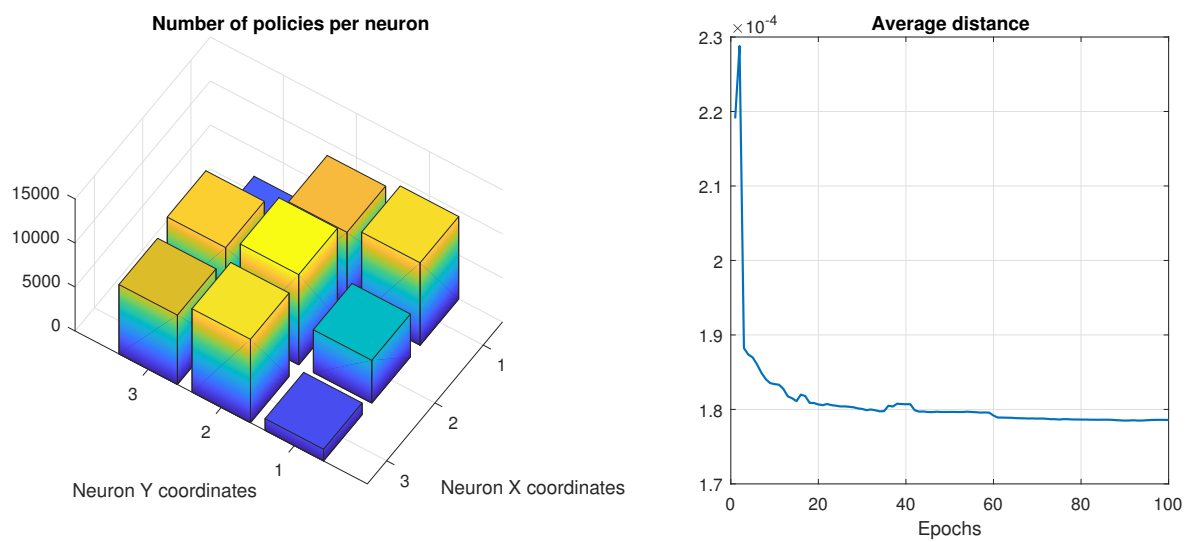

Figure 3: The left graph shows the number of policies assigned to each neuron. The right graph plots the evolution of the average distance $\frac{d^{\text {total }}}{N}$, over iterations. 


\begin{tabular}{|c|c|c|c|c|}
\hline $\begin{array}{c}\text { Neuron } \\
u\end{array}$ & $\begin{array}{c}w_{1}^{u} \times \\
\max (\text { Age })\end{array}$ & $\begin{array}{c}w_{2}^{u} \times \\
\max (\text { Veh.Age })\end{array}$ & $\bar{\lambda}_{u}$ & $\begin{array}{c}\% \text { of the } \\
\text { population }\end{array}$ \\
\hline \hline 1 & 25.77 & 4.96 & 0.0413 & 14.96 \\
\hline 2 & 27.32 & 15.34 & 0.0154 & 14.39 \\
\hline 3 & 35.27 & 40.41 & 0.0052 & 2.86 \\
\hline 4 & 41.36 & 16.85 & 0.0038 & 15.21 \\
\hline 5 & 43.22 & 4.77 & 0.0096 & 13.59 \\
\hline 6 & 51.81 & 16.58 & 0.0034 & 16.43 \\
\hline 7 & 54.06 & 4.43 & 0.0086 & 12.63 \\
\hline 8 & 56.66 & 44.60 & 0 & 2.14 \\
\hline 9 & 64.78 & 12.77 & 0.0058 & 7.79 \\
\hline
\end{tabular}

Table 2: This table reports the codebooks of neurons, the average frequency of claims for sub-populations of policies associated to each neuron, and sub-population relative sizes.

\subsection{A Bayesian regressive SOM with quantitative variables}

In this section, we aim to assign policies to groups in which we observe homogeneous of claims frequency, up to a certain level. The SOM plays then two roles. First, it segments policies based on their features. Second, it explains the claims frequency as a function of these characteristics. For this reason, the SOM is said regressive.

As in the previous section, we assume that policies are exclusively described by quantitative variables stored in a table $X$ with $N$ rows and $p$ columns. Furthermore, we define a $N \times 2$ matrix $Y$ that reports information about the number of claims, $\left(N_{i}\right)_{i=1, \ldots N}$ and the duration of the contract $\left(\nu_{i}\right)_{i=1, \ldots N}$ :

$$
Y=\left(N_{i}, \nu_{i}\right)_{i=1, \ldots, N}
$$

By nature, the number of claims $N_{i}$ is null for most of policies (693 claims for 62436 policies). The neural map is a $l \times l$ array of neurons with a matrix $C$ that contains the coordinates of nodes in $\mathbb{R}^{2}$. The codebook of the $u^{\text {th }}$ neurons is still denoted by $W_{u}=\left\{w_{1}^{u}, \ldots, w_{p}^{u}\right\}$.

We wish to construct a map of the portfolio in which policies are bundled into groups identified by a neuron. We denote by $\Omega_{u=1, \ldots, l^{2}}$ the set of insurance contracts assigned to the $u^{\text {th }}$ neuron. We assume that the number of claims caused by a policy in $\Omega_{u}$ is distributed according to a Poisson law of parameter $\lambda_{u}$. If the total exposure is $\nu^{\Omega_{u}}=\sum_{i \in \Omega^{u}} \nu_{i}$, the distribution of the total number of claims in $\Omega_{u}$, noted $N^{\Omega_{u}}$ is given by

$$
P\left(N^{\Omega_{u}}=k\right)=\frac{\left(\lambda_{u} \nu^{\Omega_{u}}\right)^{k}}{k !} e^{-\lambda_{u} \nu^{\Omega_{u}}} .
$$


The log-likelihood estimator of $\lambda_{u}$ is then equal to:

$$
\bar{\lambda}_{u}=\frac{N^{\Omega_{u}}}{\nu^{\Omega_{u}}}=\frac{\sum_{i \in \Omega^{u}} N_{i}}{\sum_{i \in \Omega^{u}} \nu_{i}} .
$$

The homogeneity of claims frequencies inside a subset $\Omega_{u}$ is measured by the realized standard deviation of $N^{\Omega_{u}}$ :

$$
d_{\Omega_{u}}\left(\bar{\lambda}_{u}\right)=\sqrt{\sum_{i \in \Omega_{u}}\left(N_{i}-\bar{\lambda}_{u} \nu_{i}\right)^{2}}
$$

and the distance between the realized frequency of the $i^{\text {th }}$ policy and average frequency for the subgroup $\Omega_{u}$ is given by

$$
d_{\Omega_{u}}\left(i, \bar{\lambda}_{u}\right)=\sqrt{\left(N_{i}-\bar{\lambda}_{u} \nu_{i}\right)^{2}} .
$$

A first idea could be to use this standard deviation to define a new metric to measure the distance between the $i^{\text {th }}$ policy and the $u^{\text {th }}$ neuron as follows:

$$
\begin{aligned}
d(u, i) & :=\left\|W_{u}-X_{i, .}\right\|_{2}+\beta d_{\Omega_{u}}\left(i, \bar{\lambda}_{u}\right) \quad u=1, \ldots, l^{2} i=1, \ldots, N \\
& =\sqrt{\sum_{k=1}^{p}\left(w_{k}^{u}-x_{i, k}\right)^{2}}+\beta \sqrt{\left(N_{i}-\bar{\lambda}_{u} \nu_{i}\right)^{2}}
\end{aligned}
$$

where $\beta$ is a weight that permits to adjust the regressive feature of the map with respect to its segmentation role. This distance could be used in equation (1) of the first step of Kohonen's algorithm 1 . Whereas $\bar{\lambda}_{u}$ would be updated at the end of each iteration, in the third step of the algorithm 1 . However, this approach is not satisfactory when applied to the insurance data. Given that the database counts only 693 claims for 62436 policies, the algorithm tends to discriminate the portfolio into two subsets: one regrouping policies with no claim and the other one gathering policies with one or more claims. To remedy to this issue, we propose a Bayesian approach.

We consider a Gamma r.v. $\Theta \sim \Gamma(\gamma, \gamma)$ and assume that conditionally to $\Theta$, the r.v. $N^{\Omega_{1}}, \ldots, N^{\Omega_{N}}$ are independent with conditional distributions:

$$
N_{u \mid \Theta} \sim \operatorname{Poi}\left(\lambda_{u} \Theta \nu^{\Omega_{u}}\right) \quad u=1, \ldots, l^{2}
$$

where $\lambda_{u}>0$ and $\gamma$ are the prior frequency of claims and a dispersion parameter. The choice of $\lambda_{u}$ and $\gamma$ is discussed at the end of this section. Under the Bayesian assumption, the coefficient of variation of the expected frequency is then $\gamma \frac{1}{\gamma^{2}}=\frac{1}{\gamma}$. Then we have the following standard result: 
Proposition 2.1. The posterior expected frequency on $\Omega_{u}$ given observations $N^{\Omega_{u}}$ is given by

$$
\mathbb{E}\left(\lambda_{u} \Theta \mid N^{\Omega_{u}}\right)=\alpha_{u} \bar{\lambda}_{u}+\left(1-\alpha_{u}\right) \lambda_{u},
$$

where $\bar{\lambda}_{u}=\frac{N^{\Omega_{u}}}{\nu^{\Omega_{u}}}=\frac{\sum_{i \in \Omega^{u}} N_{i}}{\sum_{i \in \Omega^{u}} \nu_{i}}$ and with credibility weights

$$
\alpha_{u}=\frac{\nu^{\Omega_{u}} \lambda_{u}}{\gamma+\nu^{\Omega_{u}} \lambda_{u}} \in(0,1)
$$

for $u=1, \ldots, l^{2}$.

Proof: Using the Bayes rule, we know that the probability density function of $\Theta \mid N_{\Omega^{u}}$ is equal to

$$
\begin{aligned}
& f_{\Theta}\left(\vartheta \mid N_{\Omega^{u}}=n\right)=\frac{f_{\Theta}(\vartheta) P\left(N_{\Omega^{u}}=n \mid \Theta=\vartheta\right)}{P\left(N_{\Omega^{u}}=n\right)} .
\end{aligned}
$$

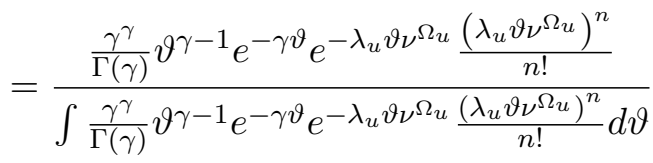

$$
\begin{aligned}
& \propto \vartheta^{\gamma+n} e^{-\left(\gamma+\lambda_{u} \nu^{\Omega_{u}}\right) \vartheta}
\end{aligned}
$$

where $\propto$ is the proportional operator. $f_{\Theta}\left(\vartheta \mid N_{\Omega^{u}}\right)$ is again the density of a Gamma distribution with the updated parameters

$$
\begin{aligned}
& \gamma^{\prime}=\gamma+N_{\Omega^{u}}, \\
& \beta^{\prime}=\gamma+\lambda_{u} \nu^{\Omega_{u}} .
\end{aligned}
$$

As $\bar{\lambda}_{u}=\frac{N^{\Omega_{u}}}{\nu^{\Omega_{u}}}=\frac{\sum_{i \in \Omega^{u}} N_{i}}{\sum_{i \in \Omega^{u}} \nu_{i}}$ then

$$
\begin{aligned}
\mathbb{E}\left(\lambda_{u} \Theta \mid N^{\Omega_{u}}\right) & =\lambda_{u} \mathbb{E}\left(\Theta \mid N^{\Omega_{u}}\right) \\
& =\lambda_{u} \frac{\gamma+N^{\Omega_{u}}}{\gamma+\lambda_{u} \nu^{\Omega_{u}}} \\
& =\lambda_{u} \frac{\gamma}{\gamma+\lambda_{u} \nu^{\Omega_{u}}}+\frac{\lambda_{u} \nu^{\Omega_{u}}}{\gamma+\lambda_{u} \nu^{\Omega_{u}}} \frac{N^{\Omega_{u}}}{\nu^{\Omega_{u}}} \\
& =\left(1-\alpha_{u}\right) \lambda_{u}+\alpha_{u} \bar{\lambda}_{u}
\end{aligned}
$$

For a given prior frequency $\lambda_{u}$ and dispersion parameter $\gamma$, the posterior mean $\mathbb{E}\left(\lambda_{u} \Theta \mid N^{\Omega_{u}}\right)$, is an estimator for the expected frequency on $\Omega_{u}$. Contrary to the empirical intensity $\bar{\lambda}_{u}$, this posterior is always strictly positive. However in practice, the prior $\lambda_{u}$ is unknown. For this reason, we opt for an empirical version of the credibility estimator (9):

$$
\mathbb{E}\left(\lambda_{u} \Theta \mid N^{\Omega_{u}}\right)=\alpha_{u} \bar{\lambda}_{u}+\left(1-\alpha_{u}\right) \bar{\lambda}
$$


where $\bar{\lambda}_{u}=\frac{N^{\Omega_{u}}}{\nu^{\Omega_{u}}}$ and $\bar{\lambda}=\frac{\sum_{u=1}^{l^{2}} N^{\Omega_{u}}}{\sum_{u=1}^{l^{2}} \nu^{\Omega_{u}}}$ is the global average claims frequency. Whereas we choose credibility weights equal to

$$
\alpha_{u}=\frac{\nu^{\Omega_{u}} \bar{\lambda}}{\gamma+\nu^{\Omega_{u}} \bar{\lambda}}
$$

A similar assumption is done in the R package "rpart", for Poisson regression trees. In the Bayesian framework, the homogeneity of claims frequency inside a subset $\Omega_{u}$ is measured by the realized standard deviation of $N^{\Omega_{u}}$, calculated with the credibility estimator:

$$
d_{\Omega_{u}}\left(\bar{\lambda}_{u}\right)=\sqrt{\sum_{i \in \Omega_{u}}\left(N_{i}-\mathbb{E}\left(\lambda_{u} \Theta \mid N^{\Omega_{u}}\right) \nu_{i}\right)^{2}},
$$

and the distance between the realized frequency of the $i^{\text {th }}$ policy and the posterior expected frequency for the subgroup $\Omega_{u}$ is given by

$$
d_{\Omega_{u}}\left(i, \bar{\lambda}_{u}\right)=\sqrt{\left(N_{i}-\mathbb{E}\left(\lambda_{u} \Theta \mid N^{\Omega_{u}}\right) \nu_{i}\right)^{2}}
$$

whereas the metric to measure the distance between the $i^{\text {th }}$ policy and the $u^{\text {th }}$ neuron in the Kohonen's algorithm becomes:

$$
\begin{aligned}
d^{r e g}(u, i) & :=\left\|W_{u}-X_{i, .}\right\|_{2}+\beta d_{\Omega_{u}}\left(i, \bar{\lambda}_{u}\right) u=1, \ldots, l^{2} i=1, \ldots, N \\
& =\sqrt{\sum_{k=1}^{p}\left(w_{k}^{u}-x_{i, k}\right)^{2}}+\beta \sqrt{\left(N_{i}-\mathbb{E}\left(\lambda_{u} \Theta \mid N^{\Omega_{u}}\right) \nu_{i}\right)^{2}}
\end{aligned}
$$

where $\beta$ is a weight adjusting the regressive feature of the map with respect to its segmentation power. The algorithm 2 summarizes the steps of the Bayesian regressive SOM. This algorithm is tested on the insurance data set provided by Ohlsson and Johansson (2010). We fit a grid of 9 neurons and the weight $\beta$ involved in the definition of the distance (13) is set to 10 . The credibility factor $\gamma$ is set to 4 . A comparison of the figure 4 that presents the regression Kohonen's map with the map of figure 3 allows us to see the impact of the Bayesian metric on the segmentation of the portfolio. In particular, the owners of a motorcycle older than 30 years are assigned to the same neuron. While a segmentation only based on owner's and vehicle ages divides this group into two subsets.

The left plot of figure 5 shows the number of policies assigned to each neurons. The two neurons associated to younger insured cover $30 \%$ of the portfolio. The right graph of figure 5 presents the evolution of the total distance between policies. Convergence is achieved after 100 iterations.

Table 3 provides detailed information about codebooks and estimated frequency of claims. 
Neurons 3 and 4 gather less $1.3 \%$ of policies but the frequency of claims associated to these risks is particularly high $(71 \%$ and $12 \%)$ compared to the average frequency $(1,06 \%)$. From a commercial point of view, insurers cannot base their tariff on claims frequencies varying so much between categories of contracts. A way to smooth frequencies of claims consists to increase the credibility parameters $\gamma$ and/or to decrease the weight $\beta$. Before developing this point, we first discuss the goodness of fit of the regression. 
Algorithm 2 Bayesian regression Kohonen's algorithm for quantitative variables.

Initialization :

Randomly attribute a vector of weights to each node of the map.

Set $\bar{\lambda}_{u}=\bar{\lambda}=\frac{\sum_{u=1}^{l^{2}} N^{\Omega_{u}}}{\sum_{u=1}^{l^{2}} \nu^{\Omega_{u}}}$.

Main procedure :

For $e=0$ to maximum epoch, $e_{\max }$

For $i=1$ to $N$

1) Find the node $u$ matching at best the features of the $i^{\text {th }}$ policy

$$
u=\arg \min _{u} d^{r e g}(u, i)
$$

This node is the BMN (best matching node).

2) Update the weight vectors of the nodes in the neighborhood of the

BMN

(including the BMN itself) by pulling them closer in $\mathbb{R}^{p}$ to the input

vector:

$$
W_{v}(e+1)=W_{v}(e)+\theta(u, v, e) \epsilon(e)\left(X_{i, .}-W_{v}(e)\right)
$$

where

$$
\begin{aligned}
\epsilon(e) & =\epsilon_{0}\left(\frac{e_{\max }-e}{e_{\max }}\right) \\
\theta(u, v, e) & =\theta_{0} \exp \left(-\frac{\left(\left\|C_{u}-C_{v}\right\|_{2}\right)^{2}}{2 \sigma(e)^{2}}\right) \\
\sigma(e) & =\sigma_{0}\left(\frac{1.2 e_{\max }-e}{e_{\max }}\right)
\end{aligned}
$$

End loop on policies, $i$

3) Calculation of the total distance $d^{\text {total }}$ between policies and best matching nodes:

$$
d^{\text {total }}=\sum_{i=1}^{N}\left\|W_{B M N(i)}-X_{i, .}\right\|_{2}+\beta d_{\Omega_{B M N(i)}}\left(i, \bar{\lambda}_{B M N(i)}\right)
$$

$1, \ldots, l^{2}$.

4) Update of $\bar{\lambda}_{u}=\frac{\sum_{i \in \Omega^{u}} N_{i}}{\sum_{i \in \Omega^{u}} \nu_{i}}$ and credibility weights $\alpha_{u}=\frac{\nu^{\Omega_{u}} \bar{\lambda}}{\gamma+\nu^{\Omega_{u}} \bar{\lambda}}$ for $u=$

End loop on epochs, $e$ 


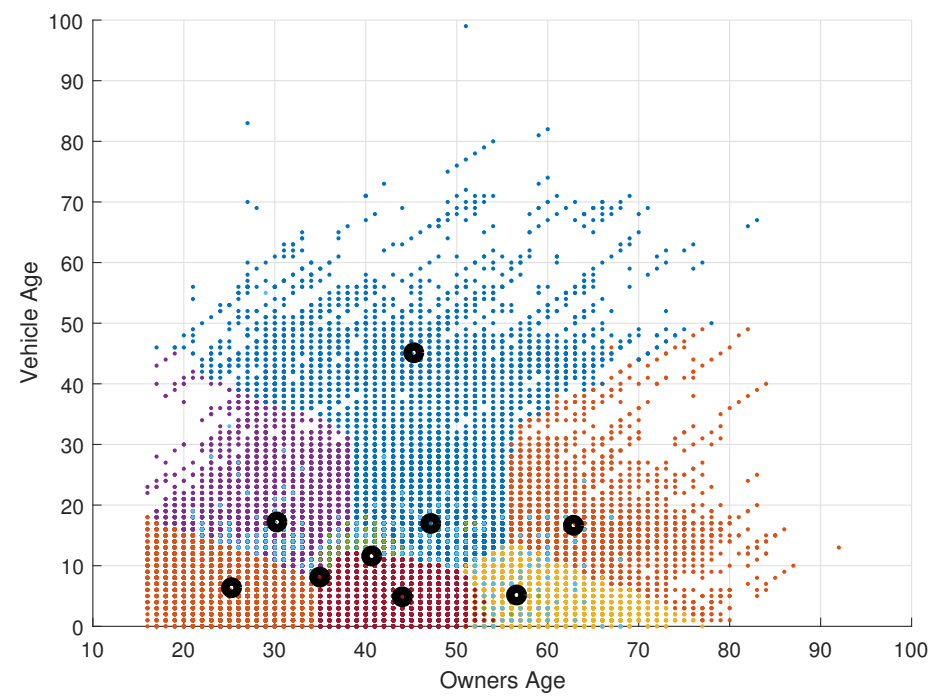

Figure 4: Regression Kohonen's map of the portfolio, with 9 neurons. The segmentation variables are the owner's age and age of the vehicle. Each policy is represented by a dot. Black dots point out the position of neurons (the coordinates are codebooks of neurons). The colors identify the area of influence of neurons.
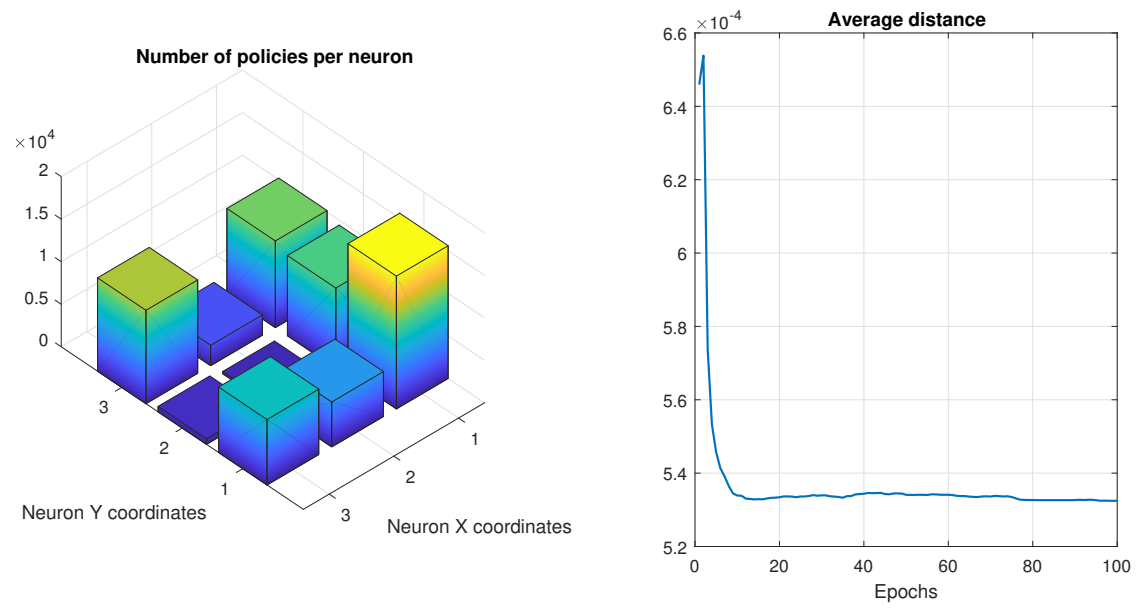

Figure 5: The left graph shows the number of policies assigned to each neuron. The right graph plots the evolution of the average distance $d^{\text {total }} / N$, over iterations. 


\begin{tabular}{|c|c|c|c|c|}
\hline $\begin{array}{c}\text { Neuron } \\
u\end{array}$ & $\begin{array}{c}w_{1}^{u} \times \\
\max (\text { Age })\end{array}$ & $\begin{array}{c}w_{2}^{u} \times \\
\max (\text { Veh. Age })\end{array}$ & $\mathbb{E}\left(\lambda_{u} \Theta \mid N^{\Omega_{u}}\right)$ & $\begin{array}{c}\% \text { of the } \\
\text { population }\end{array}$ \\
\hline \hline 1 & 26.64 & 4.41 & 0.0019 & 13.89 \\
\hline 2 & 26.96 & 15.27 & 0.0005 & 15.97 \\
\hline 3 & 34.51 & 8.02 & 0.7178 & 0.91 \\
\hline 4 & 40.50 & 6.53 & 0.1204 & 0.26 \\
\hline 5 & 41.70 & 16.63 & 0.0003 & 18.13 \\
\hline 6 & 45.58 & 44.35 & 0.0016 & 4.15 \\
\hline 7 & 47.59 & 4.43 & 0.0002 & 20.56 \\
\hline 8 & 53.27 & 16.68 & 0.003 & 16.65 \\
\hline 9 & 60.60 & 9.23 & 0.0007 & 9.48 \\
\hline
\end{tabular}

Table 3: This table reports the codebooks of neurons, the average frequency of claims for sub-populations of policies associated to each neuron. We also report the credibility estimator of the claims intensity and relative sizes of sub-populations.

The total distance $d^{\text {total }}$ both measures the quality of the regression and of the portfolio segmentation, based on the metric of equation 13. If we aim at evaluating the goodness of fit, we may be tempted to calculate the deviance. However, we will see that using credibility weights to ensure the positiveness of estimated frequencies perturbs the interpretation of this measure. The deviance is the difference between the log-likelihood of a model with many parameters as observations and the fitted model. The model counting as many parameters as observations is called the saturated model. The log-likelihood of this model, denoted by $l^{\text {saturated }}$, is computed with parameters set to empirical frequency. The saturated model has no predictive power but provides the best fit from a pure statistical point of view, given that it has the highest attainable log-likelihood. If we denote by $l$ the log-likelihood for our data, the deviance $D^{*}$ is defined as likelihood ratio test (LRT) of the model under consideration against the saturated model:

$$
D^{*}=2\left(l^{\text {saturated }}-l\right) .
$$

If $\hat{\lambda}_{i}$ is the estimate of the claims frequency for the $i^{\text {th }}$ policy forecasted by the SOM, the probability of observation $n$ claims over a period $\nu_{i}$ is equal to

$$
P\left(N_{i}=n\right)=\frac{\left(\hat{\lambda}_{i} \nu^{i}\right)^{n}}{n !} e^{-\hat{\lambda}_{i} \nu^{i}} .
$$

The contribution of the $i^{\text {th }}$ policy to the $\log$-likelihood $l$ is then equal to

$$
N_{i} \log \left(\hat{\lambda}_{i} \nu^{i}\right)-\hat{\lambda}_{i} \nu^{i}-N_{i} ! .
$$

In the saturated model, this contribution is given by

$$
\left\{\begin{array}{ll}
N_{i} \log \left(\frac{N_{i}}{\nu^{i}} \nu^{i}\right)-\frac{N_{i}}{\nu^{i}} \nu^{i}-N_{i} ! & \text { if } N_{i} \geq 1 \\
0 & \text { else if }
\end{array} .\right.
$$


The marginal deviance for the $i^{t h}$ policy is then equal to

$$
\left\{\begin{array}{ll}
2 N_{i}\left(\frac{\nu_{i}}{N_{i}} \hat{\lambda}_{i}-\log \frac{\hat{\lambda}_{i} \nu_{i}}{N_{i}}-1\right) & \text { if } N_{i} \geq 1 \\
2 \nu_{i} \hat{\lambda}_{i} & \text { if } N_{i}=0
\end{array} .\right.
$$

The total deviance is the sum of marginal contributions

$$
D^{*}=2 \sum_{i=1}^{N} N_{i}\left(\frac{\nu_{i}}{N_{i}} \hat{\lambda}_{i}-\left(\log \frac{\hat{\lambda}_{i} \nu_{i}}{N_{i}}+1\right) I_{\left\{N_{i} \geq 1\right\}}\right) .
$$

The deviance is used in statistics to compare the predictive capacity of models fitted by log-likelihood maximization. This measure is however not fully adapted to compare regressive self-organizing maps in a Bayesian set-up. This point is emphasized in table 4 that reports deviances and average distances between policies and best matching neurons, for different values of $\beta$ and credibility weights $\gamma$. As revealed by table 4 , increasing the number of neurons and then of sub-populations reduces the average distance but raises the deviance. This counter-intuitive result is the direct consequence of using credibility weights that ensure the positivity of estimated frequencies. To understand this, let us consider an extreme case in which we have as many neurons as data and when $\beta$ is small. In this case, each neuron is associated to a single policy and the frequency estimate is

$$
\mathbb{E}\left(\lambda_{i} \Theta \mid N_{i}\right)=\alpha_{i} \bar{\lambda}_{i}+\left(1-\alpha_{i}\right) \bar{\lambda},
$$

where $\bar{\lambda}_{i}=\frac{N_{i}}{\nu_{i}}$ and $\bar{\lambda}=\frac{\sum_{i=1}^{N} N_{i}}{\sum_{i=1}^{N} \nu_{i}}$. Given that the average intensity is low, $\bar{\lambda}=0.0106$, the weight $\alpha_{i}=\left(1+\frac{\gamma}{\nu_{i} \lambda}\right)^{-1}$ tends to zero and $\mathbb{E}\left(\lambda_{i} \Theta \mid N_{i}\right) \approx \bar{\lambda}$. This model has the same log-likelihood as the one obtained with a Poisson model with a single parameter. This model, called the null model, has the lowest log-likelihood and the higher deviance. Then, refining the segmentation does not necessary improve the deviance if we use credibility estimators of frequencies. This point was underlined by Wüthrich and Buser (2017) who observe the same phenomenon for regression trees. On page 83, they mention that replacing the maximum likelihood estimator (MLE) by the empirical credibility estimator has the advantage that the resulting frequency estimators are always strictly positive. The disadvantage is that errors measured by the deviance may increase by adding more splits (which turns out to refine the segmentation). This is because only the MLE minimizes the corresponding deviance statistics and maximizes the log-likelihood function, respectively.

As mentioned earlier, estimated claims frequencies obtained with a credibility weight $\gamma=4$ and reported in table 3, vary too much to build a tariff. Increasing the credibility parameters $\gamma$ and/or to decreasing the weight $\beta$ allows to smooth these frequencies. However, figures of table 4 show that this smoothing deteriorates the deviance. To get an idea to which extend large deviances are admissible, we fit a generalized linear model (GLM) to our dataset. GLM is a standard approach widely used by insurers to assess their risk. We 
regress the claims frequency with respect to six categories of owner's age: $(28 ; 31],(31 ; 35]$, $(35 ; 44],(44 ; 51],(51 ; 61]$ and $(61 ; 100]$. We fit a Poisson model with a logarithmic link function. The residual deviance for this model climbs to 6214, which is quite high compared to deviances obtained with a regressive SOM. This confirms that regressive SOMs have comparable performances to GLM. Except that it is a non-parametric and non supervised approach.

In theory, it is possible to perform $K$-fold cross validation. In practice, we face several difficulties when we implement this method. Firstly, the number of claims being small (693 claims for 62436 contracts), some bootstrapped data samples do not contain any claims. Secondly, the calibration time is quite long on a personal computer: about a half hour for 16 neurons and 100 epochs. A cross validation with 10 bootstrapped samples lasts then 5 hours.

\begin{tabular}{|ccccc|}
\hline $\begin{array}{c}\text { Neuron } \\
l^{2}\end{array}$ & $\gamma$ & $\beta$ & $D^{*}$ & $\frac{d^{\text {total }}}{N}$ \\
\hline \hline 4 & 4 & 10 & 659 & $6.535 \mathrm{e}-4$ \\
9 & 4 & 10 & 679 & $4.856 \mathrm{e}-4$ \\
16 & 4 & 10 & 694 & $4.232 \mathrm{e}-4$ \\
\hline 4 & 10 & 10 & 1097 & $6.329 \mathrm{e}-4$ \\
9 & 10 & 10 & 1252 & $5.325 \mathrm{e}-4$ \\
16 & 10 & 10 & 1167 & $4.754 \mathrm{e}-4$ \\
\hline 4 & 10 & 1 & 2160 & $7.029 \mathrm{e}-4$ \\
9 & 10 & 1 & 2347 & $5.345 \mathrm{e}-4$ \\
16 & 10 & 1 & 2519 & $4.475 \mathrm{e}-4$ \\
\hline 9 & 0 & 0 & 6087 & $1.786 \mathrm{e}-4$ \\
\hline GLM & \multicolumn{5}{|c|}{6214} \\
\hline Null model & \multicolumn{5}{|c}{} \\
\cline { 1 - 3 } & &
\end{tabular}

Table 4: This table reports deviances and average distances between policies and best matching neurons, for different values of $\beta$ and credibility weights $\gamma$. We also provide the deviance of the SOM without regression with 9 neurons, of a GLM model regressing frequencies on 6 ages categories, and the null model.

\section{Analysis of qualitative variables with a SOM}

Many features of insurance policies are described by categorical variables that cannot be handled with a classic SOM. In this section, we modify the Kohonen algorithm in order to analyze a dataset that exclusively contains this type of variables. We first propose a procedure to study the dependences between variables. In section 5, this method is combined with the Bayesian SOM to regress claims frequency on quantitative and categorical variables. 
First, we introduce the structure of data to which the algorithm is applied. The number of insurance policies is denoted by $N$. Each of these policies is described by $K$ variables which have $m_{k}$ binary modalities for $k=1, \ldots, K$. By binary, we mean that the modality $j$ of the $k^{\text {th }}$ variable is identified by an indicator variable equal to zero or one. The total number of modalities is the sum of $m_{k}: M=\sum_{k=1}^{K} m_{k}$. In further developments, we enumerate modalities from 1 to $M$. The information about the portfolio may be summarized by a $N \times M$ matrix $D=\left(d_{i, j}\right)_{i=1 \ldots N, j=1 \ldots M}$. If the $i^{\text {th }}$ policy presents the $j^{\text {th }}$ modality then $d_{i, j}=1$ and $d_{i, j}=0$ otherwise.

For example, let us assume that a policy is described by the gender $(\mathrm{M}=$ male or $\mathrm{F}=$ Female $)$ of the policyholder and by a geographic area ( $\mathrm{U}=$ urban, $\mathrm{S}=$ suburban or $\mathrm{C}=$ countryside). The number of variables and modalities are respectively $K=2, m_{1}=2$ and $m_{2}=3$. If the first and second policyholders are respectively a man living in a city and a woman living in the countryside, the two first lines of the matrix $D$ are presented in table 5 .

\begin{tabular}{|c|c|c|c|c|c|}
\hline & \multicolumn{2}{|c|}{ Gender } & \multicolumn{3}{|c|}{ Area } \\
\hline \hline Policy & M & F & U & S & C \\
\hline 1 & 1 & 0 & 1 & 0 & 0 \\
\hline 2 & 0 & 1 & 0 & 0 & 1 \\
\hline$\vdots$ & $\vdots$ & $\vdots$ & $\vdots$ & $\vdots$ & $\vdots$ \\
\hline
\end{tabular}

Table 5: Example of a disjunctive table for $K=2$ variables with respectively $m_{1}=2$, $m_{2}=3$ modalities.

The table $D$ is called a disjunctive table. In order to study the dependence between the modalities, we need to calculate the numbers $n_{i, j}$ of individuals sharing modalities $i$ and $j$, for $i, j=1, \ldots, M$. The $M \times M$ matrix $B=\left(n_{i, j}\right)_{i, j=1, \ldots, M}$ is a contingency table, called the Burt matrix containing this information. The Burt matrix is directly related to the disjunctive table as follows:

$$
B=D^{\top} D .
$$

This symmetric matrix is composed of $K \times K$ blocks $B_{k, l}$ for $k, l=1, \ldots, K$. A block $B_{k, l}$ is the contingency table that crosses the variables $k$ and $l$. The table 6 illustrates the contents of the Burt matrix related to the matrix $D$ presented in table 5 . By construction, the sum of elements of a block $B_{k, l}$ is equal to the total number of policies, $N$. The sum of $n_{i, j}$ of the same row $i$ is equal to

$$
n_{i, .}=\sum_{j=1, \ldots, M} n_{i, j}=K n_{i, i} .
$$

The Burt matrix being symmetric, we directly infer that

$$
n_{., j}=\sum_{i=1, \ldots, M} n_{i, j}=K n_{j, j} .
$$


Furthermore, blocks $B_{k, k}$ for $k=1, \ldots, K$ are diagonal matrix, whose diagonal entries are the numbers of policies who respectively present the modalities $1, \ldots, m_{k}$, for the variable $k$. In our example, we have that $n_{1,1}+n_{2,2}=N$ and $n_{3,3}+n_{4,4}+n_{5,5}=N . n_{1,1}$ and $n_{2,2}$ count the total number of men and women in the portfolio. Whereas $n_{3,3}, n_{4,4}$ and $n_{5,5}$ counts the number of policyholders living respectively in a urban, sub-urban or rural environment.

\begin{tabular}{|c|c|cc|ccc|}
\hline \multicolumn{2}{|c|}{} & \multicolumn{2}{|c|}{ Gender } & \multicolumn{3}{c|}{ Area } \\
\cline { 3 - 7 } \multicolumn{2}{c|}{} & $\mathrm{M}$ & $\mathrm{F}$ & $\mathrm{U}$ & $\mathrm{S}$ & $\mathrm{C}$ \\
\hline \multirow{2}{*}{ Gender } & $\mathrm{M}$ & $n_{1,1}$ & 0 & $n_{1,3}$ & $n_{1,4}$ & $n_{1,5}$ \\
\cline { 2 - 7 } & $\mathrm{F}$ & 0 & $n_{2,2}$ & $n_{2,3}$ & $n_{2,4}$ & $n_{2,5}$ \\
\hline \multirow{3}{*}{ Area } & $\mathrm{U}$ & $n_{3,1}$ & $n_{3,2}$ & $n_{3,3}$ & 0 & 0 \\
\cline { 2 - 7 } & $\mathrm{S}$ & $n_{4,1}$ & $n_{4,2}$ & 0 & $n_{4,4}$ & 0 \\
\cline { 2 - 7 } & $\mathrm{C}$ & $n_{5,1}$ & $n_{5,2}$ & 0 & 0 & $n_{5,5}$ \\
\hline
\end{tabular}

Table 6: Example of Burt matrix

The self-organizing map requires the definition of a distance between categorical variables. This point is discussed in the next section.

\section{A $\chi^{2}$ distance for categorical variables}

Multiple correspondence Analysis (MCA) was initially developed by Burt (1950) and enhanced by Benzécri (1973), Greenacre (1984) and Lebart et al.(1984). This technique evaluates the level of dependence between categorical variables with a $\chi^{2}$ distance. We first present this distance in the case of two variables and extend it later to the multivariate case.

\subsection{The bivariate case}

Let us first consider the case of two categorical variables, $K=2$, with $m_{1}$ and $m_{2}$ modalities. The classical MCA studies relative frequencies of crossed modalities. The table of frequency $F=\left(f_{i, j}\right)_{i, j}$ is a $m_{1} \times m_{2}$ matrix defined as follows:

$$
f_{i, j}=\frac{n_{i, j}}{N} \quad i=1, \ldots, m_{1}, j=1, \ldots, m_{2} .
$$

The marginal frequencies are equal to

$$
\begin{aligned}
& f_{i, .}=\sum_{j=1}^{m_{2}} f_{i, j}=\frac{n_{i, .}}{N} \quad i=1, \ldots, m_{1}, \\
& f_{., j}=\sum_{i}^{m_{1}} f_{i, j}=\frac{n_{., j}}{N} \quad j=1, \ldots, m_{2} .
\end{aligned}
$$


If variables are independent, the expected number of policies with modalities $i$ and $j$, is equal to $\tilde{n}_{i, j}=\frac{n_{i, .} n_{., j}}{N}$. In this case, standardized residuals $\chi_{i, j}=\frac{n_{i, j}-\tilde{n}_{i, j}}{\sqrt{\tilde{n}_{i, j}}}$ should be $N(0,1)$ random variables. If the two variables are independent, then the following statistics (called the inertia):

$$
\begin{aligned}
\chi^{2} & =\sum_{i=1}^{m_{1}} \sum_{j=1}^{m_{2}} \frac{\left(n_{i, j}-\tilde{n}_{i, j}\right)^{2}}{\tilde{n}_{i, j}} \\
& =N \sum_{i=1}^{m_{1}} \sum_{j=1}^{m_{2}} \frac{\left(f_{i, j}-f_{i, .} f_{., j}\right)^{2}}{f_{i, .} f_{., j}} \\
& =N\left(\sum_{i=1}^{m_{1}} \sum_{j=1}^{m_{2}} \frac{f_{i, j}^{2}}{f_{i, .} f_{., j}}-1\right)
\end{aligned}
$$

is a chi-square random variable with $\left(m_{1}-1\right)\left(m_{2}-1\right)$ degrees of freedom. This justifies to measure the distance between the modalities $i$ and $i^{\prime}$ of the first variable by

$$
\begin{aligned}
\chi^{2}\left(i, i^{\prime}\right) & =\sum_{j=1}^{m_{2}} \frac{1}{f_{., j}}\left(\frac{f_{i, j}}{f_{i, .}}-\frac{f_{i^{\prime}, j}}{f_{i^{\prime}, .}}\right)^{2}, \\
& =\sum_{j=1}^{m_{2}} \frac{N}{n_{., j}}\left(\frac{n_{i, j}}{n_{i, .}}-\frac{n_{i^{\prime}, j}}{n_{i^{\prime}, .}}\right)^{2} .
\end{aligned}
$$

$\chi^{2}\left(i, i^{\prime}\right)$ is the distance between the rows $i$ and $i^{\prime}$ of the matrix of frequency $F$. It may be checked that the dispersion of rows around their barycenter is equal to the inertia. Similarly, the chi-square distance between the modalities $j$ and $j^{\prime}$ of the second variable is defined by

$$
\begin{aligned}
\chi^{2}\left(j, j^{\prime}\right) & =\sum_{i=1}^{m_{1}} \frac{1}{f_{i, .}}\left(\frac{f_{i, j}}{f_{., j}}-\frac{f_{i, j^{\prime}}}{f_{., j^{\prime}}}\right)^{2} \\
& =\sum_{i=1}^{m_{1}} \frac{N}{n_{i, .}}\left(\frac{n_{i, j}}{n_{., j}}-\frac{n_{i, j^{\prime}}}{n_{., j^{\prime}}}\right)^{2} .
\end{aligned}
$$

This measures the distance between columns of the matrix $F$. In the algorithm of the self-organizing map, we prefer to evaluate distances between rows and columns with the Euclidian distance. It is then convenient to replace the frequencies $f_{i, j}$ by weighted values $f_{i, j}^{W}:$

$$
f_{i, j}^{W}:=\frac{f_{i, j}}{\sqrt{f_{i, .} f_{., j}}}=\frac{n_{i, j}}{\sqrt{n_{i, .} n_{., j}}} \quad i=1, \ldots, m_{1} j=1, \ldots, m_{2}
$$


The distances between rows $\left(i, i^{\prime}\right)$ and columns $\left(j, j^{\prime}\right)$ simplify as follows:

$$
\begin{aligned}
\chi^{2}\left(i, i^{\prime}\right) & =\sum_{j=1}^{m_{2}}\left(f_{i, j}^{W}-f_{i^{\prime}, j}^{W}\right)^{2} . \\
\chi^{2}\left(j, j^{\prime}\right) & =\sum_{i=1}^{m_{1}}\left(f_{i, j}^{W}-f_{i, j^{\prime}}^{W}\right)^{2} .
\end{aligned}
$$

Finally, notice that the matrix $F^{W}=\left(f_{i, j}^{W}\right)_{i=1, \ldots, m_{1}, j=1, \ldots, m_{2}}$ is symmetric by construction.

\subsection{The multivariate case}

In this section, we extend the notion of $\chi^{2}$ distance between categorical variables to the multivariate case $(K>2)$. Remember that the Burt table, $B=\left(n_{i, j}\right)_{i, j=1, \ldots, M}$, is a contingency table. This symmetric matrix is composed of $K \times K$ blocks $B_{k, l}$ and $B_{k, l}$ is the contingency table that crosses the variables $k$ and $l$. It is then natural to extend the definition of distance (15) between rows $i$ and $i^{\prime}$ of the Burt matrix as follows:

$$
\chi^{2}\left(i, i^{\prime}\right)=\sum_{j=1}^{M} \frac{N}{n_{., j}}\left(\frac{n_{i, j}}{n_{i, .}}-\frac{n_{i^{\prime}, j}}{n_{i^{\prime}, .}}\right)^{2} \quad i, i^{\prime} \in\{1, \ldots, M\} .
$$

Similarly, the chi-square distance between columns $j$ and $j^{\prime}$ of the Burt matrix is defined by

$$
\chi^{2}\left(j, j^{\prime}\right)=\sum_{i=1}^{M} \frac{N}{n_{i, .}}\left(\frac{n_{i, j}}{n_{., j}}-\frac{n_{i, j^{\prime}}}{n_{., j^{\prime}}}\right)^{2} \quad j, j^{\prime} \in\{1, \ldots, M\} .
$$

As mentioned in the previous section, we prefer to evaluate distances with the Euclidian distance. We replace then the elements of the Burt matrix $n_{i, j}$ by weighted values $n_{i, j}^{W}$ :

$$
n_{i, j}^{W}:=\frac{n_{i, j}}{\sqrt{n_{i, .} n_{., j}}} \quad i, j=1, \ldots, M
$$

Given that the sums $n_{i, .}=K n_{i, i}$ and $n_{., j}=K n_{j, j}$, we have that .

$$
n_{i, j}^{W}:=\frac{n_{i, j}}{K \sqrt{n_{i, i} n_{j, j}}} \quad i, j=1, \ldots, M
$$

If $C$ is the diagonal matrix $C=\operatorname{diag}\left(n_{11}^{-\frac{1}{2}} \cdot . n_{M M}^{-\frac{1}{2}}\right)$ then the weighted Burt matrix is denoted by $B^{W}$ :

$$
B^{W}=\frac{1}{K} C B C
$$


The distances between rows $\left(i, i^{\prime}\right)$ and columns $\left(j, j^{\prime}\right)$ of the Burt matrix becomes in this case

$$
\begin{aligned}
\chi^{2}\left(i, i^{\prime}\right) & =\sum_{j=1}^{m_{2}}\left(n_{i, j}^{W}-n_{i^{\prime}, j}^{W}\right)^{2}, \\
\chi^{2}\left(j, j^{\prime}\right) & =\sum_{i=1}^{m_{1}}\left(n_{i, j}^{W}-n_{i, j^{\prime}}^{W}\right)^{2} .
\end{aligned}
$$

\subsection{Application to insurance data}

In the previous section, we have seen that each modality of categorical variables is represented by a line of the weighted Burt matrix. A line of this matrix defines a point in a space with $M$ dimensions. The level of dependence between modalities $i$ and $j$ is measured by the Euclidian distance between two points with coordinates contained in the $i^{\text {th }}$ and $j^{t h}$ lines of $B^{W}$. We can then apply the Kohonen's algorithm 1 directly to the weighted Burt matrix in order to study the relations between categorical variables. We consider the categorical variables described in table 1 to which we add a new categorical variable representative of the owner's age. We consider three modalities for the owner's age, that are constructed according to the rule reported in table 7 .

\begin{tabular}{|c|c|}
\hline \multicolumn{2}{|c|}{ Discretized Age categories } \\
\hline Modality & Value \\
\hline \hline Young & Owners Age $<35$ years old \\
\hline Mature & 35 years old $\leq$ Owners Age $<55$ years old \\
\hline Old & 55 years old $\leq$ Owners Age \\
\hline
\end{tabular}

Table 7: Table of modalities for the categorical variable representative of the owner's age.

We fit a $4 \times 4 \mathrm{SOM}$ to the matrix $B^{W}$. The number of epochs is set to $e_{\max }=1000$. The initial codebooks are equal to randomly drawn lines of $B^{W}$. The parameters of equations (3), (4) and (5) for the update of codebooks are: $\epsilon_{0}=0.01, \theta_{0}=1$ and $\sigma_{0}=$ 0.10 . The right graph of figure 6 confirms that the network is trained after 850 iterations. Whereas the left plot shows that 6 neurons are not assigned to any modality. One neuron is coupled to 4 modalities and two neurons explain 3 modalities. Details about the pooling of modalities are provided in table 8 . This reveals that the recurrent insured profile is a mature man, living in the countryside and owning a vehicle of class 3 . We also learn that a majority of young insureds lives in small cities whereas insured women are living in a urban environment and drive a motorcycle of class 4 . The older category of policyholders drives a vehicle of class 2 whereas people living in northern cities or Gotland mainly owns powerful motorcycle (class 7). This analysis reveals that SOMs allows detecting the most common associations of modalities. With this information, we can draw an identikit image of the average policy. However, we haven't regressed the claims frequency on categorical variables yet. 

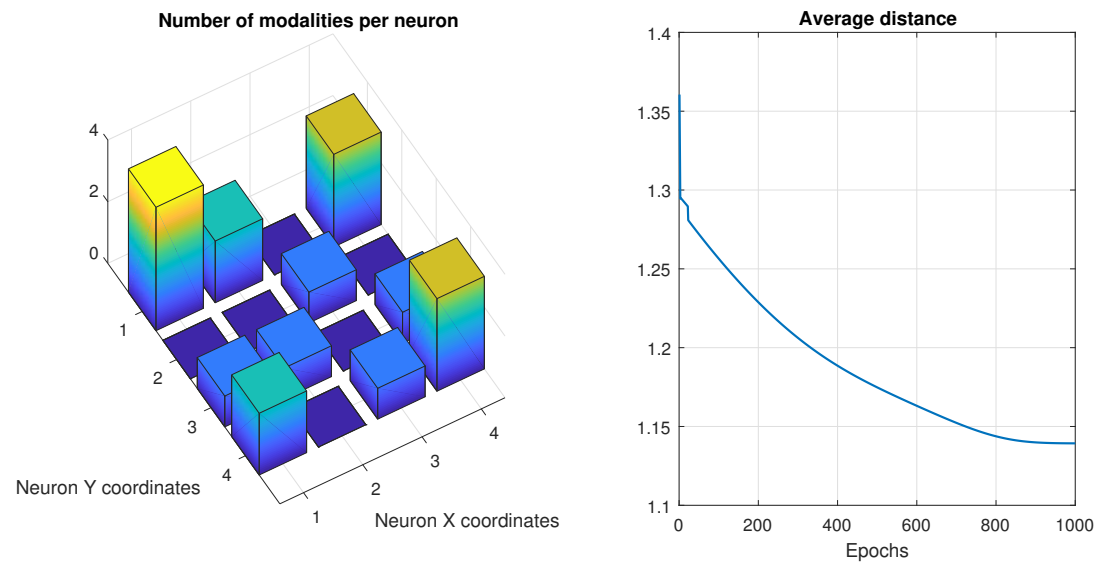

Figure 6: The left graph shows the number of modalities assigned to each neuron. The right graph plots the evolution of the average distance over iterations.

\begin{tabular}{|ccc||ccc|}
\hline Neuron $u$ & Modalities & $\begin{array}{c}\text { Marginal } \\
\text { frequency }\end{array}$ & Neuron $u$ & Modalities & $\begin{array}{c}\text { Marginal } \\
\text { frequency }\end{array}$ \\
\hline 1 & Mature & 0.006 & 9 & Class 5 & 0.011 \\
\cline { 4 - 6 } & Men & 0.011 & 10 & Suburban & 0.016 \\
\cline { 5 - 6 } & Countryside & 0.006 & 12 & North. village & 0.006 \\
\cline { 5 - 6 } & Class 3 & 0.008 & 13 & Old & 0.005 \\
\cline { 5 - 6 } 2 & Young & 0.027 & 13 & Class 2 & 0.014 \\
\hline \multirow{2nnnyy}{*}{4} & Small city & 0.010 & 15 & Class 6 & 0.020 \\
\hline & Women & 0.009 & 16 & North. city & 0.006 \\
& Urban & 0.029 & 16 & Gotland & 0.004 \\
\hline 7 & Class 4 & 0.008 & 16 & Class 7 & 0.018 \\
\hline
\end{tabular}

Table 8: Groups of modalities per neuron. We also report the average claims frequency for each of these modalities.

\section{A regressive SOM with quantitative and categorical vari- ables}

In this section, we aim to explain the claims frequency by categorical and quantitative variables. We intend to segment the portfolio by assigning each policy to a neuron in the SOM using all the available information. To achieve this goal, we combine the Bayesian framework of section 2 with the SOM for categorical variables of section 4 . As in previous sections, the $N$ policies are described by $p$ quantitative and $K$ categorical variables. The categorical variables have $m_{k}$ binary modalities for $k=1, \ldots, K$. The total number of 
modalities is denoted $M=\sum_{k=1}^{K} m_{k}$. The information about the portfolio is summarized by the $M \times M$ weighted Burt matrix $B^{W}$. Whereas the feature of each policies are reported in the $N \times M$ disjunctive table $D$. The quantitative variables stored in a table $X$ with $N$ rows and $l$ columns. The $N \times 2$ matrix $Y$ contains the number of claims, $\left(N_{i}\right)_{i=1, \ldots N}$ and the duration of the contract $\left(\nu_{i}\right)_{i=1, \ldots N}$.

The neural map is a $l \times l$ array of neurons with a matrix $C$ that contains the coordinates of nodes in $[0,1] \times[0,1]$. Neurons are equally spaced on this pavement. The codebook of the $u^{\text {th }}$ neurons is now composed of a $p$-vector

$$
Q_{u}=\left(q_{1}^{u}, \ldots, q_{p}^{u}\right)
$$

for quantitative variables and of a $M$ vector

$$
W_{u}=\left(w_{1}^{u}, \ldots, w_{M}^{u}\right)
$$

for qualitative variables. The distance between the quantitative variables of the $i^{\text {th }}$ policy and the neuron is measured by the 2 norm : $\left\|Q_{u}-X_{i, .}\right\|_{2}$. The modalities of categorical variables are represented by points in a space with $M$ dimensions and their coordinates are contained in weighted Burt matrix. It is then natural to measure the distance between the modalities of the $i^{\text {th }}$ policy and the neuron by the following norm:

$$
\left\|W_{u}-1_{K}^{\top} D_{i, .}^{\top} B^{W} / K\right\|_{2},
$$

where $1_{K}$ is a unit vector of dimension $K$ and $D_{i, .}$ is the $i^{\text {th }}$ line of the disjunctive table. The quantity (20) is the distance between the neuron represented by a point of coordinates $W_{u}$ in $\mathbb{R}^{M}$ and the barycenter of the cloud of points corresponding to modalities characterizing the $i^{\text {th }}$ policy.

As in previous section we denote by $\Omega_{u=1, \ldots, l^{2}}$ the set of insurance contracts assigned to the $u^{t h}$ neuron. The credibility estimator of the expected number of claims caused by a policy in $\Omega_{u}$ is equal to:

$$
\mathbb{E}\left(\lambda_{u} \Theta \mid N^{\Omega_{u}}\right)=\alpha_{u} \bar{\lambda}_{u}+\left(1-\alpha_{u}\right) \bar{\lambda}
$$

where $\bar{\lambda}_{u}=\frac{N^{\Omega_{u}}}{\nu^{\Omega_{u}}}$ and $\bar{\lambda}=\frac{\sum_{u=1}^{l^{2}} N^{\Omega_{u}}}{\sum_{u=1}^{l^{2}} \nu^{\Omega_{u}}}$ is the global average claims frequency. The credibility weights are proportional to the total exposure in $\Omega_{u}$ :

$$
\alpha_{u}=\frac{\nu^{\Omega_{u}} \bar{\lambda}}{\gamma+\nu^{\Omega_{u}} \bar{\lambda}} \quad u=1, \ldots, l^{2} .
$$

The homogeneity of claims frequency inside a subset $\Omega_{u}$ is measured by the realized standard deviation of $N^{\Omega_{u}}$ :

$$
d_{\Omega_{u}}\left(\bar{\lambda}_{u}\right)=\sqrt{\sum_{i \in \Omega_{u}}\left(N_{i}-\mathbb{E}\left(\lambda_{u} \Theta \mid N^{\Omega_{u}}\right) \nu_{i}\right)^{2}}
$$


and the distance between the realized frequency of the $i^{t h}$ policy and expected posterior distribution for the subgroup $\Omega_{u}$ is given by

$$
d_{\Omega_{u}}\left(i, \bar{\lambda}_{u}\right)=\sqrt{\left(N_{i}-\mathbb{E}\left(\lambda_{u} \Theta \mid N^{\Omega_{u}}\right) \nu_{i}\right)^{2}} .
$$

We use this standard deviation to define a new metric to measure the distance between the $i^{t h}$ policy and the $u^{\text {th }}$ neuron:

$$
\begin{aligned}
d^{r e g}(u, i) & :=\left\|Q_{u}-X_{i, .}\right\|_{2}+\beta_{1}\left\|W_{u}-1_{K}^{\top} D_{i, .}^{\top} B^{W} / K\right\|_{2} \\
& +\beta_{2} d_{\Omega_{u}}\left(i, \bar{\lambda}_{u}\right) u=1, \ldots, l^{2} i=1, \ldots, N
\end{aligned}
$$

where $\beta_{1}$ and $\beta_{2}$ are weights that permits to adjust the regressive feature of the map with respect to its segmentation role. The algorithm 3 presents the procedure to build the Bayesian regressive SOM with this distance.

\subsection{Application to insurance data}

We fit a $5 \times 5$ regression SOM to our insurance data set. We consider the three categorical variables described in table 1 and the two quantitative variables: owner's age and vehicle age. Quantitative variables are scaled on the interval $[0,1]$ as done in equations (7). We perform 3000 iterations. On a personal computer, the calibration takes between 4 and 6 hours for 9 up to 25 neurons. Parameters for the update of codebooks are: $\epsilon_{0}=0.01$, $\theta_{0}=1$ and $\sigma_{0}=0.10$. The weights $\beta_{1}$ and $\beta_{2}$ involved in the definition of the distance (21) are set to one. In order to smooth the claims frequencies for different type of policies, we choose a credibility parameter $\gamma=10$. The left plot of figure 7 presents the allocation of policies among the neural grid. We will later in this section that the neuron gathering the highest number of policies ( $8.2 \%$ of the dataset), regroups mainly mature men, living in the countryside and driving a class 3 vehicle. In section 2.2 , we have identified that this set of features is dominant in the portfolio. The right graph of figure 7 shows the evolution of the average distance between the features of a policy and the codebooks of the best matching neuron $\left(\frac{1}{N} d^{\text {total }}\right)$. Convergence is achieved after 2500 iterations.

As mentioned in sub-section 2.2, the deviance is not necessary adapted to compare regressive self-organizing maps in a Bayesian set-up. This point is confirmed in table 9, segmenting the portfolio does not necessary improve the deviance due to the use of credibility weights to estimate claims frequencies. We compare the performance the SOM to GLM by fitting a Poisson model, with a logarithmic link function. We use as covariates: the gender, the area, the class of the vehicle, six categories of owner's age $((28 ; 31],(31 ; 35]$, $(35 ; 44],(44 ; 51],(51 ; 61]$ and $(61 ; 100])$ and two categories of vehicle age $((0 ; 10],(10 ; 100])$ . The deviances for the GLM and null models are respectively equal to 5775 and 6648 . In term of deviances, the SOM provides a less good fit than a GLM. However this conclusion must be nuanced given that SOMs are totally unsupervised and non parametric methods. The deviance of SOM may also be adjusted by modifying the credibility parameter $\gamma$. 
$\overline{\text { Algorithm } 3 \text { Bayesian Regression Kohonen's algorithm for quantitative and categorical }}$ variables.

\section{Initialization :}

Randomly attribute a vector of weights to each node of the map.

Set $\bar{\lambda}_{u}=\bar{\lambda}=\frac{\sum_{u=1}^{l^{2}} N^{\Omega_{u}}}{\sum_{u=1}^{l^{2}} \nu^{\Omega_{u}}}$.

Main procedure :

For $e=0$ to maximum epoch, $e_{\max }$

For $i=1$ to $N$

1) Find the node $u$ matching at best the features of the $i^{\text {th }}$ policy

$$
u=\arg \min _{u} d^{r e g}(u, i)
$$

This node is the BMN (best matching node).

2) Update the weight vectors of the nodes in the neighborhood of the

BMN

(including the BMN itself) by pulling them closer in $\mathbb{R}^{p}$ to the input

vector:

$$
\begin{gathered}
Q_{v}(e+1)=Q_{v}(e)+\theta(u, v, e) \epsilon(e)\left(X_{i, .}-Q_{v}(e)\right) \\
W_{v}(e+1)=W_{v}(e)+\theta(u, v, e) \epsilon(e)\left(1_{K}^{\top} D_{i, .}^{\top} B^{W} / K-W_{\nu}\right)
\end{gathered}
$$

where

$$
\begin{aligned}
\epsilon(e) & =\epsilon_{0}\left(\frac{e_{\max }-e}{e_{\max }}\right) \\
\theta(u, v, e) & =\theta_{0} \exp \left(-\frac{\left(\left\|C_{u}-C_{v}\right\|_{2}\right)^{2}}{2 \sigma(e)^{2}}\right) \\
\sigma(e) & =\sigma_{0}\left(\frac{1.2 e_{\max }-e}{e_{\max }}\right)
\end{aligned}
$$

End loop on policies, $i$

3) Calculation of the total distance $d^{\text {total }}$ between policies and best matching nodes:

$$
\begin{aligned}
d^{\text {total }} & =\sum_{i=1}^{N}\left\|Q_{B M N(i)}-X_{i, .}\right\|_{2}+\beta_{1}\left\|W_{B M N(i)}-1_{K}^{\top} D_{i, .}^{\top} B^{W} / K\right\|_{2} \\
& +\beta_{2} d_{\Omega_{B M N(i)}}\left(i, \bar{\lambda}_{B M N(i)}\right)
\end{aligned}
$$

4) Update of $\bar{\lambda}_{u}=\frac{\sum_{i \in \Omega^{u}} N_{i}}{\sum_{i \in \Omega^{u}} \nu_{i}}$ for $u=1, \ldots, l^{2}$.

End loop on epochs, $e$ 

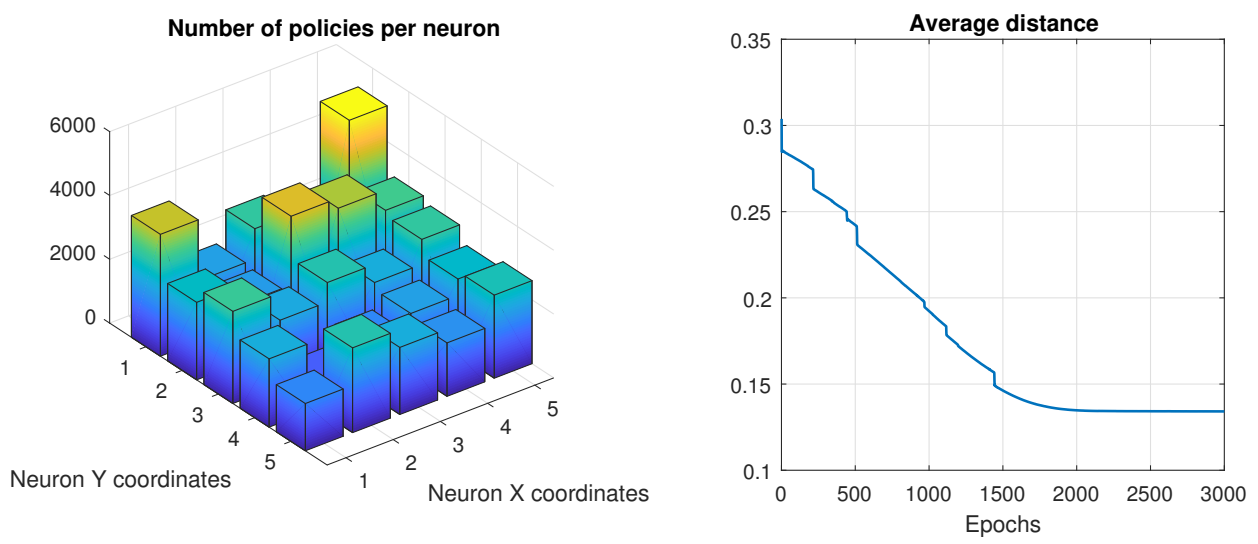

Figure 7: The left graph shows the number of policies assigned to each neuron. The right graph plots the evolution of the average distance over iterations.

Furthermore, as shown in sub-section 2.2, a model with a small deviance does not necessary provides a satisfactory segmentation of the portfolio.

\begin{tabular}{|c|c|c|}
\hline & Deviance & $\frac{d^{\text {total }}}{N}$ \\
\hline \hline 9 neurons & 6865 & 0.3089 \\
\hline 16 neurons & 6161 & 0.2568 \\
\hline 25 neurons & 6256 & 0.1341 \\
\hline
\end{tabular}

Table 9: Deviances of regressive SOMs with 9, 16 and 25 neurons.

The most interesting information for an insurer is reported in table 10 that presents the dominant modalities associated to each neuron. The $j^{\text {th }}$ modality of the $k^{\text {th }}$ qualitative variable is dominant for the $u^{\text {th }}$ neuron, if it minimizes the distance between the codebook $W_{u}$ and the lines of $B^{W}$ corresponding to the $m_{k}$ categories of the $k^{t h}$ variable:

$$
j=\arg \min _{v \in\left\{1, . ., m_{k}\right\}}\left\|W_{u}-B_{\sum_{d=1}^{k-1} m_{d}+v, .}^{W}\right\|_{2} .
$$

For quantitative variables, the average owner's and vehicle age of contracts assigned to the $u^{t h}$ neuron are respectively equal to $q_{1}^{u} \times \max (A g e)$ and $q_{2}^{u} \times \max (V e h$. Age $)$. Among the dominant profiles, we retrieve categories (Men, Countryside, Class 3), (Men, Countryside, Class 4) and (Men, Urban, Class 4). Policies assigned to these three neurons represents respectively $8.2 \%, 6.39 \%$ and $6.12 \%$ of the population. Only five neurons are associated to women. The reason is that the population of female drivers is under represented in the portfolio (9843 women on 62436 contracts). 


\begin{tabular}{lllllllll}
\hline$u$ & Gender & Area & Type & Age & $\begin{array}{l}\text { Veh. } \\
\text { Age }\end{array}$ & Freq. & $\begin{array}{l}\text { Real } \\
\text { Freq }\end{array}$ & $\begin{array}{l}\text { of } \\
\text { pop. }\end{array}$ \\
\hline 1 & Men & Ctryside & Class3 & 23.87 & 8.26 & 0.0421 & 0.066 & 3.37 \\
2 & Women & Suburban & Class3 & 27.32 & 11.31 & 0.0123 & 0.0135 & 3.43 \\
3 & Men & Smlcity & Class6 & 38.94 & 9.76 & 0.0161 & 0.0194 & 2.38 \\
4 & Men & Ctryside & Class6 & 40.51 & 10.33 & 0.0106 & 0.0106 & 4.25 \\
5 & Women & Ctryside & Class5 & 40.65 & 13.11 & 0.0063 & 0.0038 & 3.27 \\
6 & Men & Suburban & Class5 & 41.56 & 11.04 & 0.0167 & 0.0199 & 2.97 \\
7 & Men & Urban & Class4 & 41.62 & 10.4 & 0.0226 & 0.0267 & 6.12 \\
8 & Men & Smlcity & Class5 & 42.42 & 11.98 & 0.0125 & 0.0133 & 3.34 \\
9 & Men & Suburban & Class4 & 42.58 & 11.27 & 0.0124 & 0.0134 & 3.05 \\
10 & Men & Urban & Class3 & 42.62 & 10.23 & 0.0211 & 0.0254 & 3.89 \\
11 & Men & Ctryside & Class5 & 43.01 & 12.03 & 0.0059 & 0.0052 & 5.88 \\
12 & Women & Ctryside & Class3 & 43.22 & 9.78 & 0.0076 & 0.0065 & 4.62 \\
13 & Men & Northcity & Class3 & 43.7 & 10.95 & 0.0075 & 0.0053 & 2.93 \\
14 & Men & Ctryside & Class4 & 43.87 & 12.71 & 0.0057 & 0.0049 & 6.39 \\
15 & Men & Suburban & Class6 & 44.04 & 10.22 & 0.0216 & 0.027 & 3.79 \\
16 & Men & Suburban & Class3 & 44.73 & 10.81 & 0.0091 & 0.0086 & 4.58 \\
17 & Men & Smlcity & Class4 & 44.88 & 11.24 & 0.0093 & 0.0089 & 4.59 \\
18 & Men & Ctryside & Class2 & 44.89 & 14.67 & 0.0101 & 0.0099 & 3.19 \\
19 & Men & Smlcity & Class3 & 45.04 & 11.07 & 0.0068 & 0.0057 & 4.86 \\
20 & Men & Ctryside & Class1 & 45.41 & 23.9 & 0.007 & 0.0054 & 4.17 \\
21 & Men & Ctryside & Class3 & 45.47 & 12.49 & 0.0038 & 0.0031 & 8.2 \\
22 & Women & Ctryside & Class6 & 45.67 & 10.12 & 0.0159 & 0.0237 & 1.42 \\
23 & Men & Smlcity & Class1 & 46.55 & 38.12 & 0.0058 & 0.0023 & 2.68 \\
24 & Women & Smlcity & Class5 & 47.41 & 11.29 & 0.0081 & 0.0058 & 2.37 \\
25 & Men & Northvlge & Class3 & 47.69 & 12.53 & 0.0059 & 0.0038 & 4.26 \\
\hline & & & & & & & &
\end{tabular}

Table 10: This table reports the dominant features for each neurons, estimated claims frequency and percentages of the insured population assigned to each neuron.

The SOM does not only segment the portfolio, it also allows regressing the claims frequency on explanatory variables. We create a matrix $X$ filled with owners and vehicle ages of policies for which we want to assess the claim frequency. Next, we construct the disjunctive table $D$ containing the qualitative modalities of these policies. Finally, the best matching neurons are those that minimize the distance to the data, as defined in equation (21). In order to assess the predictive capacity of our model, we plot in figure 8 forecast claims frequencies for different risk profiles and owner's ages. The vehicle age is constant and set to one year. 

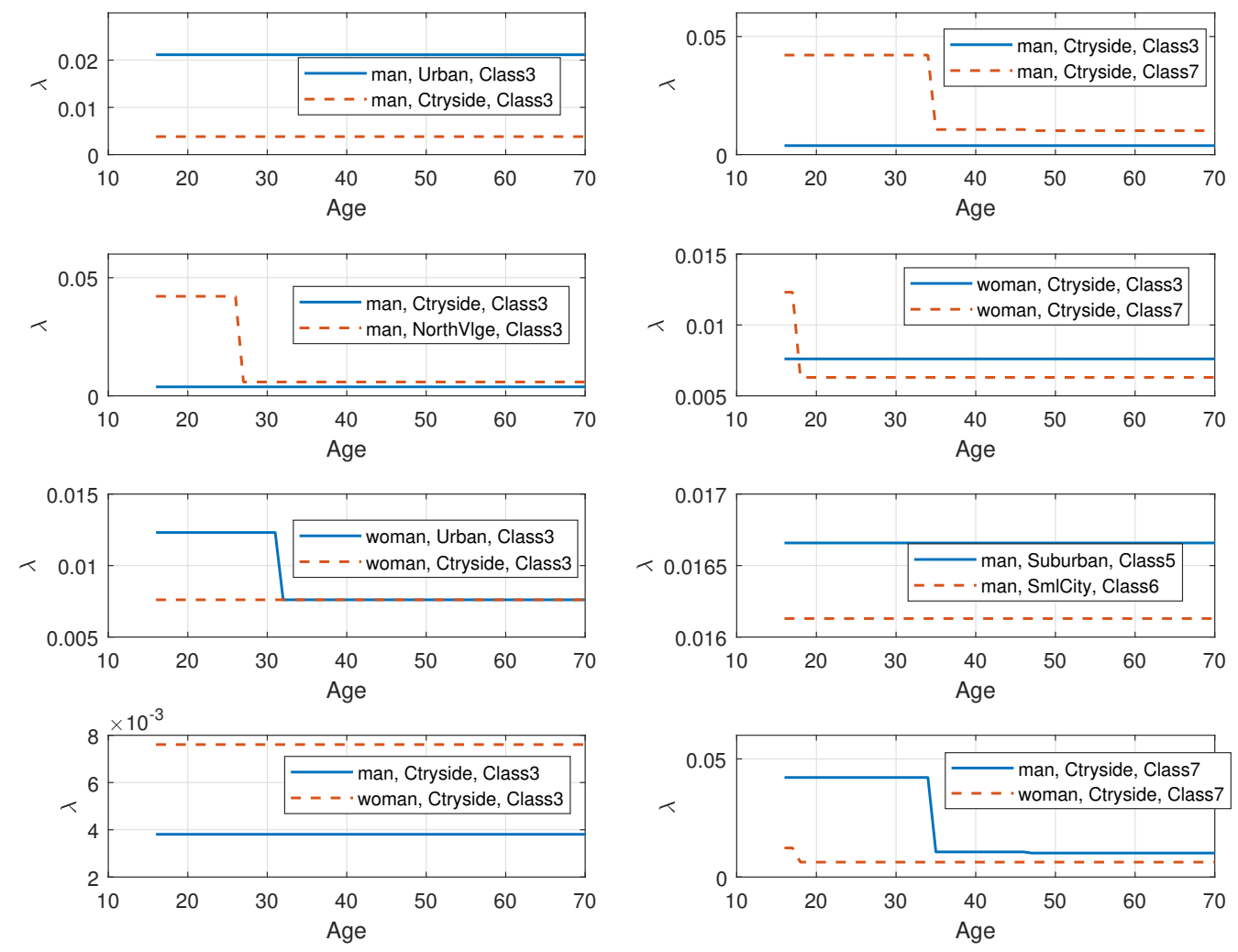

Figure 8: Regressed claims frequency for different profiles of insured.

We draw several interesting conclusions from figure 4. Men living in a urban environment have a higher probability of accident with a class 3 motorcycle than men living in the countryside driving the same vehicle. The claims frequency for men from northern villages is higher up to 28 years old. Contrary to men, female drivers living in a city and in the countryside share the same claims frequency for ages above 33 years. Female drivers of a class 3 motorcycle in the countryside have a higher probability of reporting a claim than men of the same area. More surprising, women older than 18 years, driving the most powerful vehicle (class 7) have a smaller claims frequency than class 3 drivers. However, these results must be nuanced given that women are under represented in the portfolio ( $15.76 \%$ of the data set). Male drivers of a class 7 motorcycle in the countryside have a higher claims frequency than class 3 drivers but the frequency falls above 35 years old. On average, we also expect less claims for persons living in a small city than insureds in a suburban environment. Finally, female drivers of powerful vehicle cause less accident than male drivers, in the countryside. For men, this frequency falls around 35 years old 
whereas for women, the claims frequency falls around 19 years old. These results confirm that SOM may be use for segmenting policyholders and forecasting the claims frequency.

\section{Conclusions}

We demonstrate the usefulness of self-organizing maps for analysing insurance data. Selforganizing maps are unsupervised algorithms and do not need any a priori knowledge about the dependence structure between explanatory variables. Then, they may be used to detect collinearity and to design modalities of covariates feeding a multilayer perceptron or a generalized linear model. We present four variants of the Kohonen's algorithm. The first one allows to pool policyholders based on quantitative explanatory variables. The second algorithm allows regressing the claims frequency on quantitative variables. Due to the low frequency of claims, we propose a Bayesian model to avoid a null probability of claims occurrence for some categories of insureds. With this model, the deviance, which is a traditional measure of the goodness of fit, is not necessary reduced when segmentation increases due to credibility weights. The third algorithm studies the dependence between categorical variables. It uses a $\chi^{2}$ distance similar to the one used in a multiple correspondence analysis. This efficient procedure allows drawing an identikit image of dominant variables in the portfolio. The fourth procedure both segments policyholders and regresses claims frequency on quantitative and categorical variables. This approach helps to understand the geography of risks by assigning to a dominant profile of insureds to each neuron of the SOM.

\section{References}

[1] Burt, C. 1950. The factorial analysis of qualitative data, British Journal of Psychology, Vol 3, 166-185.

[2] Benzécri, J.P. 1973. L'analyse des données, T2, l'analyse des correspondances, Dunod, Paris.

[3] Brockett P., Cooper W. , Golden L., Pitaktong U 1994. A Neural Network Method for Obtaining an Early Warning of Insurer Insolvency. The Journal of Risk and Insurance, Vol 61(3), pp. 402-424

[4] Brockett P., Xia X., Derrig. R. 1998. Using Kohonen's Self-Organizing Feature Map to Uncover Automobile Bodily Injury Claims Fraud. Journal of Risk and Insurance, Vol 65(2), pp. 245-274

[5] Brockett P., Golden L., Jang J., Yang C. 2006. A Comparison of Neural Network, Statistical Methods, and Variable Choice for Life Insurers' Financial Distress Prediction. Journal of Risk and Insurance, Vol 73(3), pp 397-419. 
[6] Cottrell M., Ibbou S., Letrémy P. 2004. SOM-based algorithms for qualitative variables. Neural networks, Vol 17, pp 1149-1167.

[7] Greenacre, M.J. 1984. Theory and Applications of Correspondence Analysis, London, Academic Press.

[8] Hainaut D., 2017. A neural network analyzer for mortality forecast. Forthcoming in the ASTIN Bulletin.

[9] Huysmans J., Baesens B., Vanthienen J., Van Gestel T. 2006. Failure prediction with self organizing maps. Expert Systems with Applications, Vol 30 (3), pp 479-487.

[10] Hertz, J., Krogh, A., Palmer, R.G. 1991. Introduction to the Theory of Neural Computation, Reading, MA : Addison-Wesley.

[11] Kohonen T. 1982. Self-Organized Formation of Topologically Correct Feature Maps. Biological Cybernetics. Vol 43 (1): 59-69

[12] Lebart, L., Morineau, A., Warwick, K.M. 1984. Multivariate Descriptive Statistical Analysis: Correspondence Analysis and Related Techniques for Large Matrices, Wiley.

[13] Ogunnaike R.M. Si D. 2017. Prediction of Insurance Claim Severity Loss Using Regression Models. Proceedings of the 13th Conference on Machine Learning and Data Mining. Springer eds. pp 233-247.

[14] Ohlsson E., Johansson B. 2010. Non-Life Insurance Pricing with Generalized Linear Models. Springer

[15] Rosenblatt F. 1958. The perceptron: a probabilistic model, for information storage and organization in the brain. Psychological Review, Vol 65(6), pp 386-408.

[16] Sakthivel K. M. and Rajitha C.S. 2017. A Comparative Study of Zero-inflated, Hurdle Models with Artificial Neural Network in Claim Count Modeling. International Journal of Statistics and Systems. Vol 12(2), pp. 265-276

[17] Wütrich M., Buser C. 2017. "Data Analytics for Non-Life Insurance Pricing". Lectures notes, available on https://papers.ssrn.com/sol3/papers.cfm?abstract_id $=2870308$

[18] Viaene, S., Derrig, R. A., Baesens, B. and Dedene, G. 2002. A Comparison of Stateof-the-Art Classification Techniques for Expert Automobile Insurance Claim Fraud Detection. Journal of Risk and Insurance, Vol 69, pp. 373-421

\section{Acknowledgment}

I gratefully acknowledges the BNP Cardiff Chair "Data Analytics and Models for Insurance" for its financial support. I also thank Michel Denuit from the UCL for his constructive advices. 